\title{
Automatización de un Secador Convectivo de Aire Caliente para fines de Docencia en Ingeniería de Alimentos
}

Jorge A. Saavedra ${ }^{1}$, Luis A. Puente ${ }^{2}$, Guillermo A. González ${ }^{3}$ e Iván B. Camousseigt ${ }^{3}$

(1) Pontificia Universidad Católica de Valparaíso, Escuela de Alimentos, Av. Brasil 2950,

Valparaíso-Chile (email: jorge.saavedra@ucv.cl)

Universidad Tecnológica Metropolitana, (2) Departamento de Biotecnología, (3) Departamento de

Electricidad, Avenida Dieciocho № 161, Santiago-Chile

\begin{abstract}
Resumen
El presente trabajo describe el diseño y montaje de un dispositivo experimental para la automatización de un secador de aire caliente con flujo paralelo en co-corriente para realizar estudios de secado. El desarrollo del trabajo se dividió en dos actividades principales: (i) diseño del sistema de automatización y (ii) la validación de los datos entregados por el equipo. Se obtuvo un dispositivo capaz de registrar las variables del proceso de secado y de la imagen de la muestra deshidratada en tiempo real, como así mismo transmitir dicha información a través de Internet. Los datos procesados por el sistema automático comparados con experiencias manuales tradicionales (no automatizadas) se validaron estadísticamente. Se concluye finalmente que la propuesta es viable, fiable y atractiva para el usuario por lo que facilita el proceso de enseñanza-aprendizaje en un área de especial importancia en Ingeniería de Alimentos.
\end{abstract}

\section{Automation of a Convective Hot Air Dryer for Teaching in Food Engineering}

\begin{abstract}
This paper describes the design and installation of an automated experimental device applied to a parallel co-current hot air dryer to perform drying studies in the field of food engineering. The development of this work was divided into two main activities: (i) design of the automation system and (ii) validation of automatically collected data. A device capable of collecting and registering real time data and images from drying processes, and transmitting them through Internet was obtained. The data processed by the automatic device was statistically validated. It is concluded that the proposed automation device is feasible, reliable and attractive for the users, facilitating the teachinglearning process in an important area of Food Engineering.
\end{abstract}

Keywords: hot air drying, automation, remote monitoring, remote laboratory 


\section{INTRODUCCIÓN}

Hoy en día, los avances tecnológicos en las redes de comunicación avanzan a una velocidad vertiginosa, lo que permite mejorar la calidad del servicio respecto del acceso a Internet y al ancho de banda disponible, situación que responde a la fuerte demanda de este tipo de servicios existente en la actualidad. La amplia disponibilidad y la capacidad de estas nuevas facilidades de comunicación, combinadas con el rápido desarrollo de las tecnologías Web y la generalización de componentes de uso común para la adquisición de datos y el control de procesos industriales, permiten incorporar estos recursos a procesos de enseñanza-aprendizaje en docencia universitaria evitando la asistencia a los laboratorios tradicionales y la vez permitiendo la accesibilidad a otras instalaciones remotas.

Una de los principales problemas asociado al uso de un laboratorio tradicional (LT) radica en la limitación de recursos en personas, tiempo y espacios restringidos, principalmente debido a la masificación de la educación superior y a los inherentes problemas presupuestarios asociados. La situación tradicional requiere de la presencia física del estudiante y la supervisión del profesor. Una solución a esta situación la constituye la implementación de laboratorios virtuales y remotos (LV/LR), de modo de facilitar la realización de experiencias a un mayor número de alumnos, incluso en ubicaciones remotas, es decir en un laboratorio virtual se podría manipular y observar el comportamiento de fenómenos y modelos físicos en cualquier momento del día y ubicación física.

En este contexto se puede decir que la enseñanza de la Ingeniería de los Alimentos posee una complejidad inherente considerando que los alimentos son considerados como matrices complejas (Harper et al., 2003, Fito et al., 2007), y en especial aquellos fenómenos de transferencia de materia y energía que están involucrados en operaciones de procesado tal como la Deshidratación, lo que se traduce en cierto grado de dificultad en el proceso de enseñanza-aprendizaje de los alumnos (Welti-Chanes et al., 2002; Tapia et al., 2005).

Tradicionalmente el estudio de la operación de deshidratación se realiza a través de determinaciones de masa del producto durante el tiempo de tal manera de realizar posteriormente las correspondientes gráficas de secado. Estos experimentos pueden resultar tediosos, por la extensión que implican, el registro manual de los datos y el evidente error humano que se incorpora a la información generada (Fito et al., 2001). Una alternativa a la experimentación con fines docentes ha sido la simulación computacional de procesos, los que, según Ogot et al. (2003), constituyen una herramienta útil y practica a la hora de representar la problemática asociada a los fenómenos de transporte para efectos ingenieriles, no obstante en términos del proceso de enseñanza-aprendizaje posee una limitación: la simulación reproduce artificialmente el fenómeno mediante modelos matemáticos que no siempre son un buen reflejo del fenómeno real, incluso careciendo de experimentación propiamente tal, lo que en rigor no es el fenómeno en si (Simpson et al., 2004).

Frente a esto Palou et al. (2003), promueven la implementación de interfaces informáticas en los laboratorios como herramientas de automatización, computación y de comunicación vía Internet de modo de que alumnos ubicados físicamente fuera de las instalaciones puedan acceder al fenómeno real a través de una interfaz informática

En este contexto, el presente trabajo consiste en el diseño y montaje de un dispositivo automático para la realización de estudios de secado, capaz de registrar datos de las variables asociadas al proceso de deshidratación (evolución de la humedad, temperaturas de bulbo seco y húmedo, velocidad del aire, entre otros) como mejora de la calidad del proceso de enseñanza-aprendizaje en la Ingeniería de alimentos.

\section{MATERIALES Y METODOS}

Para el diseño del Sistema de Automatización se procedió a intervenir un Secador de Aire Caliente de Flujo paralelo - co-corriente disponible en la Planta Piloto de la Escuela de Ingeniería en Industria Alimentaria de la Universidad Tecnológica Metropolitana. El equipo poseía las siguientes características: Cámara de Deshidratación de 0,70 m de largo x 0,45 m de alto y 0,45 m de ancho, 
totalizando un volumen de 0,1418 $\mathrm{m}^{3}$ y un área de flujo de paso de 0,2025 $\mathrm{m}^{2}$. Así mismo el equipo disponía de un ventilador monofásico de 0,2 HP capaz de generar velocidades de aire comprendidas en el rango 2,0 - 6,0 m/s. El equipo permite operar a temperaturas de trabajo entre los $25^{\circ} \mathrm{C}$ y $75^{\circ} \mathrm{C}$, para lo cual cuenta con 2 resistencias eléctricas disipadoras de calor de $850 \mathrm{~W}$ cada una, además el equipo soporta alturas del lecho de entre 1,0 cm. hasta $2,2 \mathrm{~cm}$. (ver Fig. 1a y 1b).

El planteamiento de la automatización considero prescindir de toda intervención manual del operador, de modo de eliminar errores por manipulación al momento de realizar experiencias de deshidratación durante laboratorios prácticos. Para ello se consideraron los requisitos básicos:

- Registro automatizado de datos del proceso de secado.

- Interfaz visual y amistosa de comunicación con el usuario, compatible con procesos de docencia e investigación.

- Posibilidad de transmisión de información y monitoreo hacia puntos remotos vía Internet.

A tal efecto, se considero el diseño e incorporación de instrumental automático para la captura y procesamiento de los datos obtenidos en las experiencias practicas. Esto implico el siguiente equipamiento: Anemómetro LCA-30, de 0,25 - 30 m/s (TSI, Inc.) y Balanza Mod. PB-1502-L, de $1510 \mathrm{~g} \times 0,01 \mathrm{~g}$ (Precisión Hispana), ambos con interfaz de comunicación RS-232. Se incluyen dos sensores de temperatura PT-100 clase B DIN 43760 / IEC 751, una Cámara Digital VideoCam Express II (Genius, Inc.). La información generada por este instrumental es recopilada para su procesamiento en un Computador Personal genérico (Pentium 4).

Adicionalmente se incorporó un sistema de registro de imágenes (mediante la cámara digital incluida en el equipamiento) de modo poder transmitir en directo los experimentos que se realicen (junto a la totalidad de información generada) hacia un terminal remoto (otro Computador Personal) vía Internet. En forma complementaria dicha información podría ser utilizada para realizar procesamiento de imagen con la finalidad de estudiar fenómenos asociados a la deshidratación como el cambio de color y volumen-forma (shrinkage). La figura 1 presenta el dispositivo experimental y sus componentes.
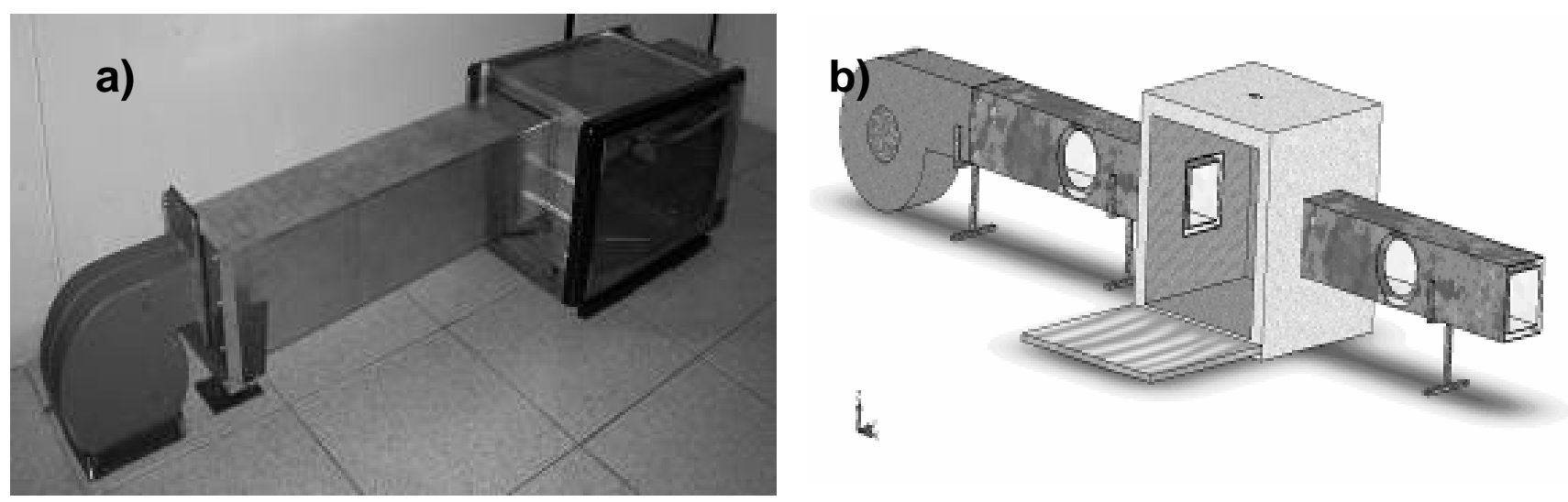

Fig. 1: a) Secador piloto b) modelo 3D secador piloto

La totalidad de los datos capturados durante el proceso experimental son procesados mediante un Circuito de Control basado en un Microcontrolador PIC18F452, el cual fue complementado con un software de interfaz gráfica, programado en lenguaje de alto nivel, Labview. Esta aplicación tiene la función de capturar, procesar y traducir los datos en información en tiempo real (humedad del producto en base seca, temperatura de bulbo húmedo, temperatura de bulbo seco, humedad relativa y la velocidad de secado). Todos los datos visualizados en la interfaz gráfica son almacenados en una detallada base de datos Excel ${ }^{\circledR}$ para su posterior análisis (Figura 2). 


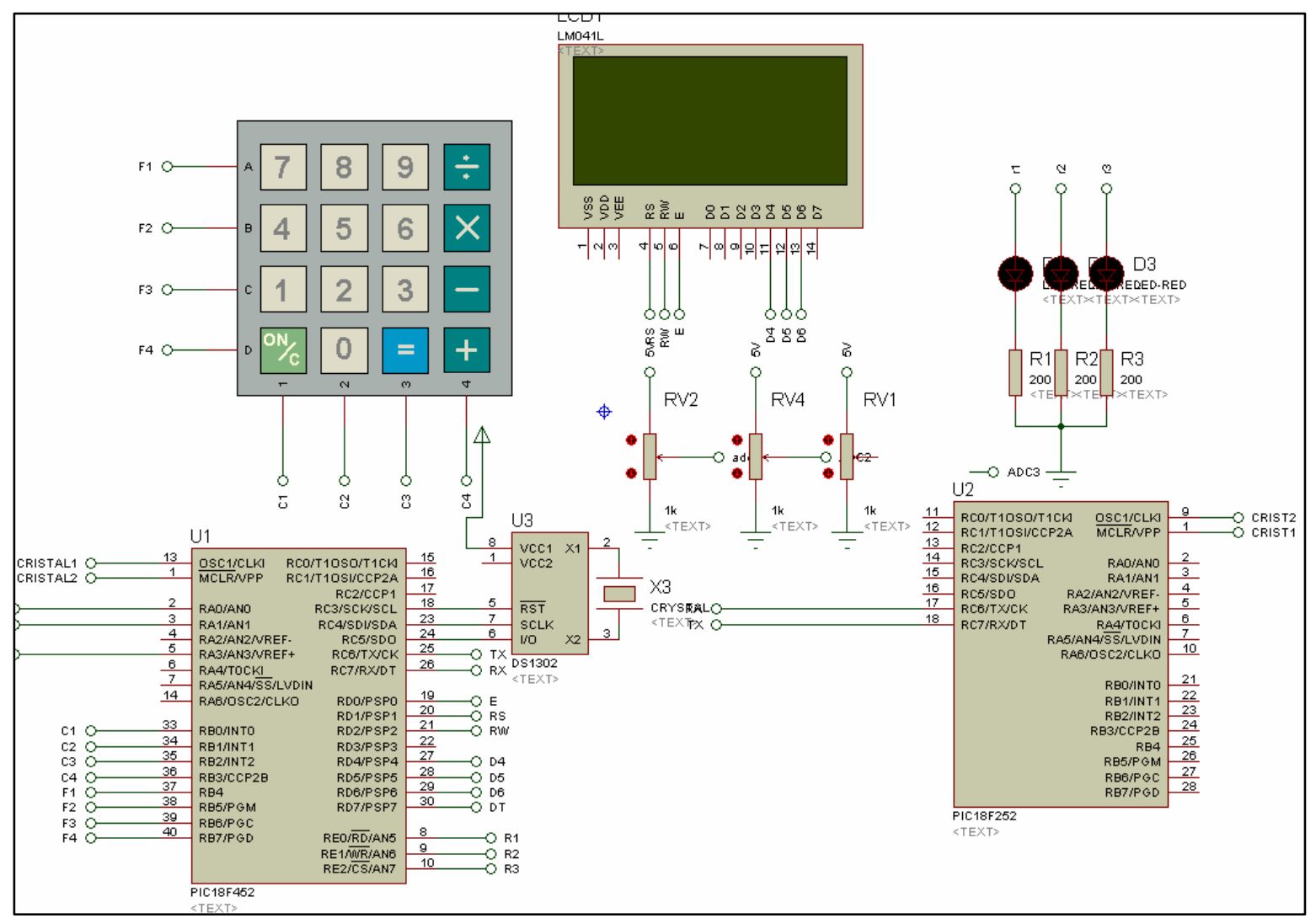

Fig. 2: Diagrama simplificado del Circuito del Sistema de Control.

La interfaz de comunicación visual (Figura 3) se puede operar tanto a nivel local como remoto en un PC que tenga acceso a Internet alámbrico o inalámbrico. Esta interfaz entrega la siguiente información: ventana de visualización de video (1), control de captura de imagen (2), nombre del archivo de imagen (formato JPG) (3), gráfico en tiempo real de los datos de humedad versus tiempo (4), temperaturas de bulbo húmedo y seco (5), velocidad del aire de secado (6), velocidad de secado calculada a partir de la derivada del grafico de humedad versus tiempo (7).

\section{RESULTADOS Y DISCUSIÓN}

Con el objetivo de comparar y validar el dispositivo automatizado respecto de la metodología tradicional, se procedió a realizar pruebas de secado de cilindros de manzana Granny Smith de 2.2 $\mathrm{cm}$. de diámetro y $1 \mathrm{~cm}$. considerando tres temperaturas de trabajo $\left(45,55\right.$ y $\left.65^{\circ} \mathrm{C}\right)$ y una velocidad de aire de de $2,4 \mathrm{~m} / \mathrm{s}$ hasta alcanzar una humedad aproximada del $17 \%$ b.h. $(0.210 \mathrm{~g} \mathrm{agua} / \mathrm{g}$ m.s.). La humedad inicial de la muestra en fresco se determinó por diferencia de peso en estufa Shell Lab Forced Air Oven 1370 Fx, hasta peso constante. (O.A.C. 920.151., 2005.). El valor fue de $86.18 \%$ b.h. (6.240 g agua/g m.s.).

Los experimentos de secado se llevaron a cabo por triplicado utilizando el dispositivo experimental operando desde la Planta Piloto de la Universidad Tecnológica Metropolitana en Santiago de Chile y fueron visualizados vía Internet en los Laboratorios de Procesos de la Pontificia Universidad Católica de Valparaíso, donde un grupo de alumnos (usuarios) pudo observar y registrar la información experimental en forma remota a través de un PC en tiempo real.

En forma paralela se realizaron experiencias en forma manual (no automatizadas) bajo las mismas condiciones experimentales y en triplicado para cada temperatura de trabajo, según la metodología planteada por Vega et al. (2005). 


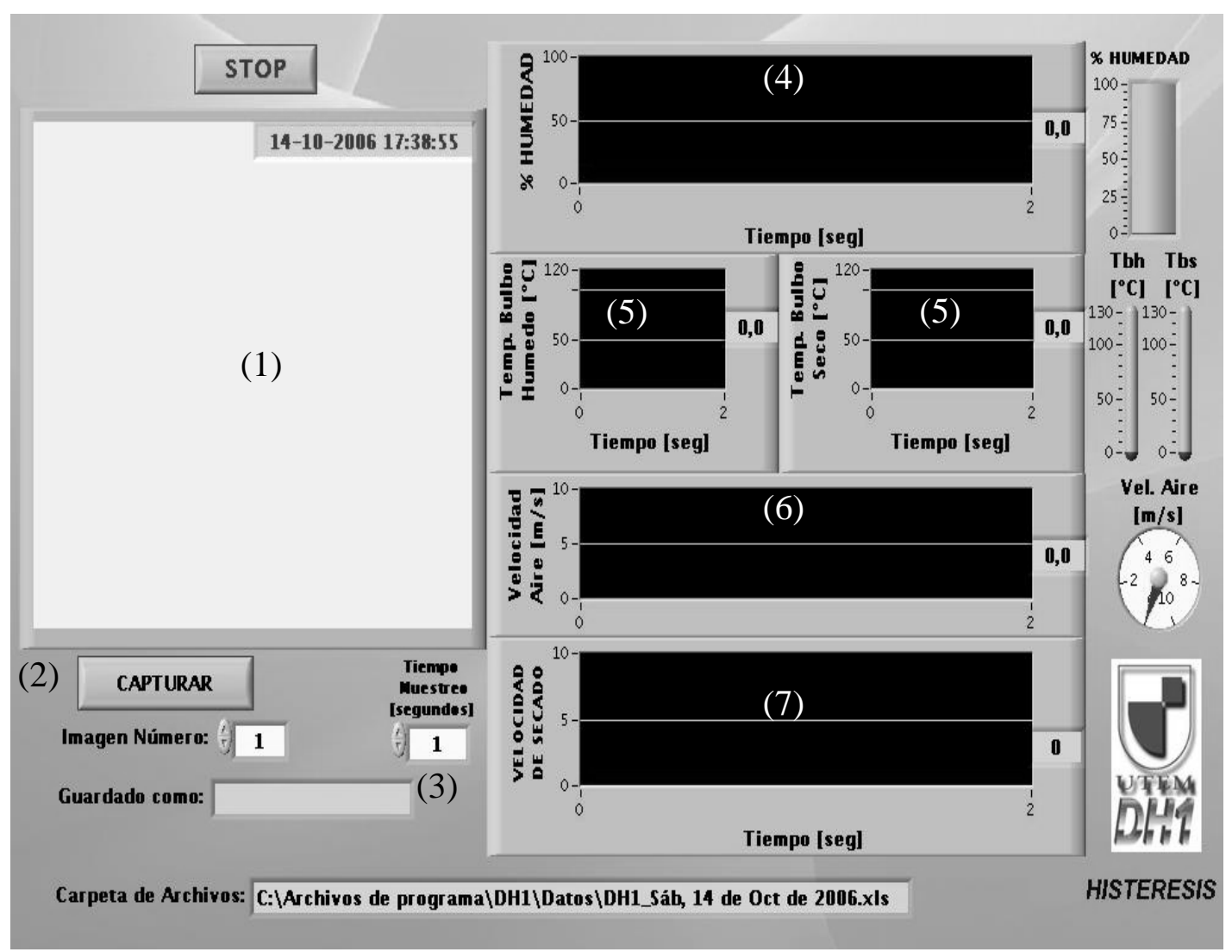

Fig. 3: Interfaz de comunicación con el usuario

Posteriormente se construyeron las Curvas de Secado para las tres temperaturas de trabajo utilizadas $\left(45,55\right.$ y $\left.65^{\circ} \mathrm{C}\right)$, las que se presentan en la figura 4. Con el objetivo de homogeneizar la comparación de las diferentes experiencias, el contenido de humedad promedio se expreso como una fracción del contenido inicial de agua presente en las muestras expresado en base seca $\left(\mathrm{X}_{0}\right)$.

Se puede apreciar claramente la diferencia entre las curvas, básicamente atribuible a los menores tiempos de secado que se obtienen al aumentar la temperatura, específicamente para 45,55 y $65^{\circ} \mathrm{C}$, se lograron tiempos de secado de 1080, 540 y 240 min., respectivamente. Así mismo se comprueba que para todas las temperaturas ensayadas, las curvas de secado presentan una tendencia exponencial decreciente.

Los criterios estadísticos utilizados para evaluar la bondad de ajuste entre los valores obtenidos con el dispositivo automático respecto de los datos obtenidos por metodología analítica fueron: el coeficiente de determinación, $\mathrm{R}^{2}$ (ec. 1), la suma de los errores cuadrados (ec. 2) y la raíz media de la suma de los errores cuadrados, RMSE (ec. 3). Estos resultados se muestran en la Tabla 1.

$$
\begin{aligned}
& R^{2}=\frac{s c E}{s c G}=\frac{\sum_{i=1}^{n}\left(\hat{y}_{i}-\bar{y}\right)^{2}}{\left.\sum_{i=1}^{n} y_{i}-\bar{y}\right)^{2}} \\
& S S E=\frac{1}{N} \sum_{i=1}^{N}\left(Y_{c i}-Y_{e i}\right)^{2} \\
& R M S E=\sqrt{\frac{1}{N} \sum_{i=1}^{N}\left(Y_{c i}-Y_{e i}\right)^{2}}
\end{aligned}
$$




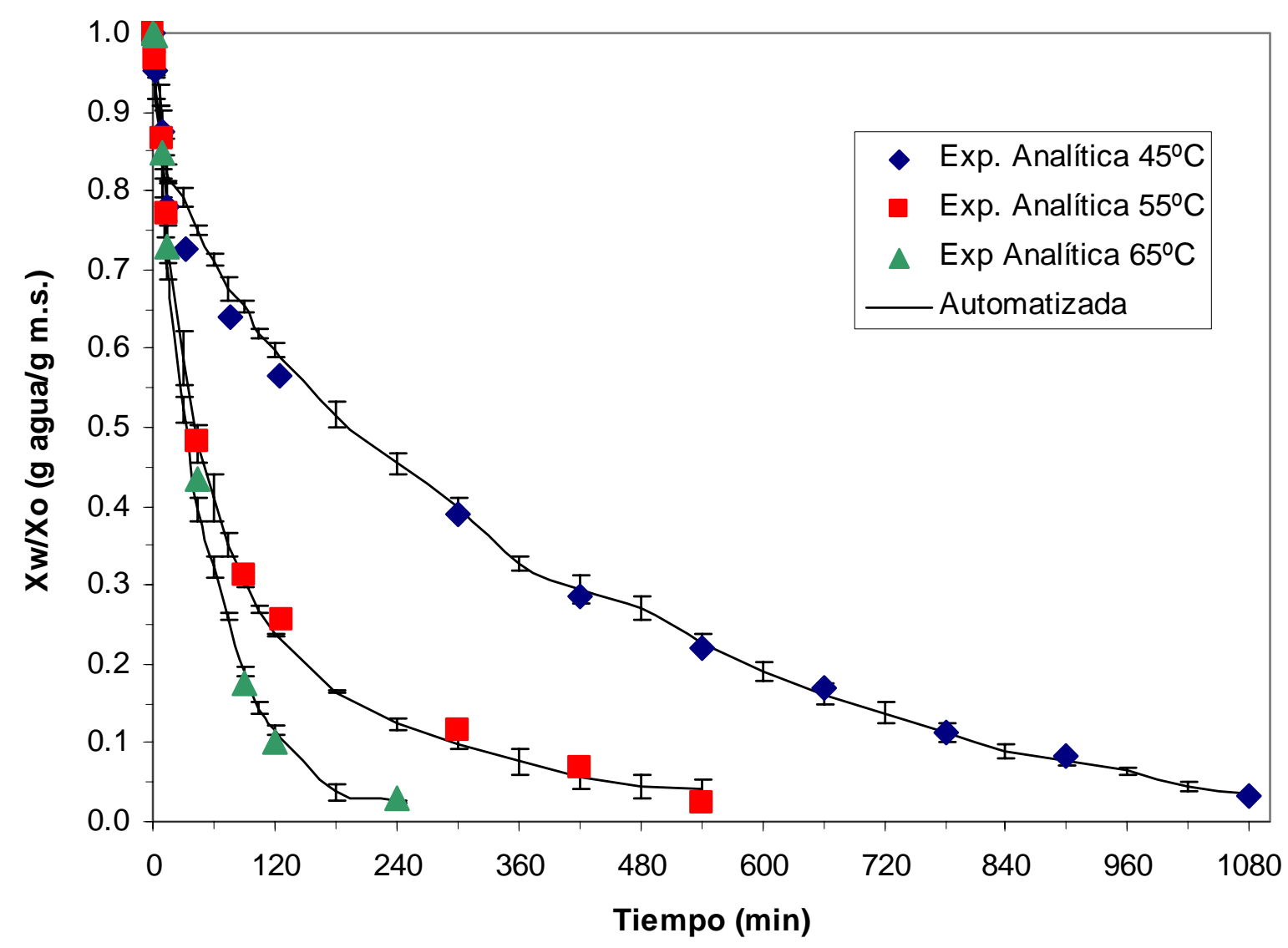

Fig. 4: Curvas de secado experimentales analíticas y automatizadas para cilindros de manzana a tres temperaturas

Tabla 1: Pruebas estadísticas para la validación de los datos para cada temperatura de secado

\begin{tabular}{|c|c|c|c|}
\hline$T\left({ }^{\circ} \mathrm{C}\right)$ & $\mathrm{R}^{2}$ & $\mathrm{SSE}$ & $\mathrm{RMSE}$ \\
\hline 45 & 0.9977 & 0.0086 & 0.0933 \\
\hline 55 & 0.9972 & 0.0092 & 0.0969 \\
\hline 65 & 0.9976 & 0.0101 & 0.1041 \\
\hline
\end{tabular}

Del análisis de los estadísticos mostrados en la tabla 1 se observa que para las tres temperaturas ensayadas los valores de $\mathrm{R}^{2}$ fueron superiores a 0.99 , lo que se interpreta para efectos de validación que las variables correlacionadas presentan un alto grado de explicación entre si. Además se puede indicar que la temperatura que presento el mejor ajuste fue $45^{\circ} \mathrm{C}$, posiblemente debido a que dicho ensayo tomo mas tiempo en lograr la humedad final, con lo cual se generó una mayor cantidad de datos, lo que facilitó el ajuste estadístico. En relación a los valores de SSE y RMSE, estos fueron en general inferiores a 0.1 , lo cual se puede considerar como aceptable considerando el fin docente del equipamiento en cuestión.

Como complemento para verificar los periodos de secado (y solo a modo informativo), se procedió a construir la curva de velocidad de secado automatizada para cada temperatura (figura 5). Se puede apreciar que las curvas para las tres temperaturas ensayadas no presentan periodo de velocidad constante, lo que concuerda con el comportamiento reportado en la literatura para manzanas y otros vegetales como zanahorias, papas, frambuesas, arvejas, tomates y mango, entre otros (Nieto et al., 1998, 2001; Simal et al., 1998; Wang et al., 2007). 


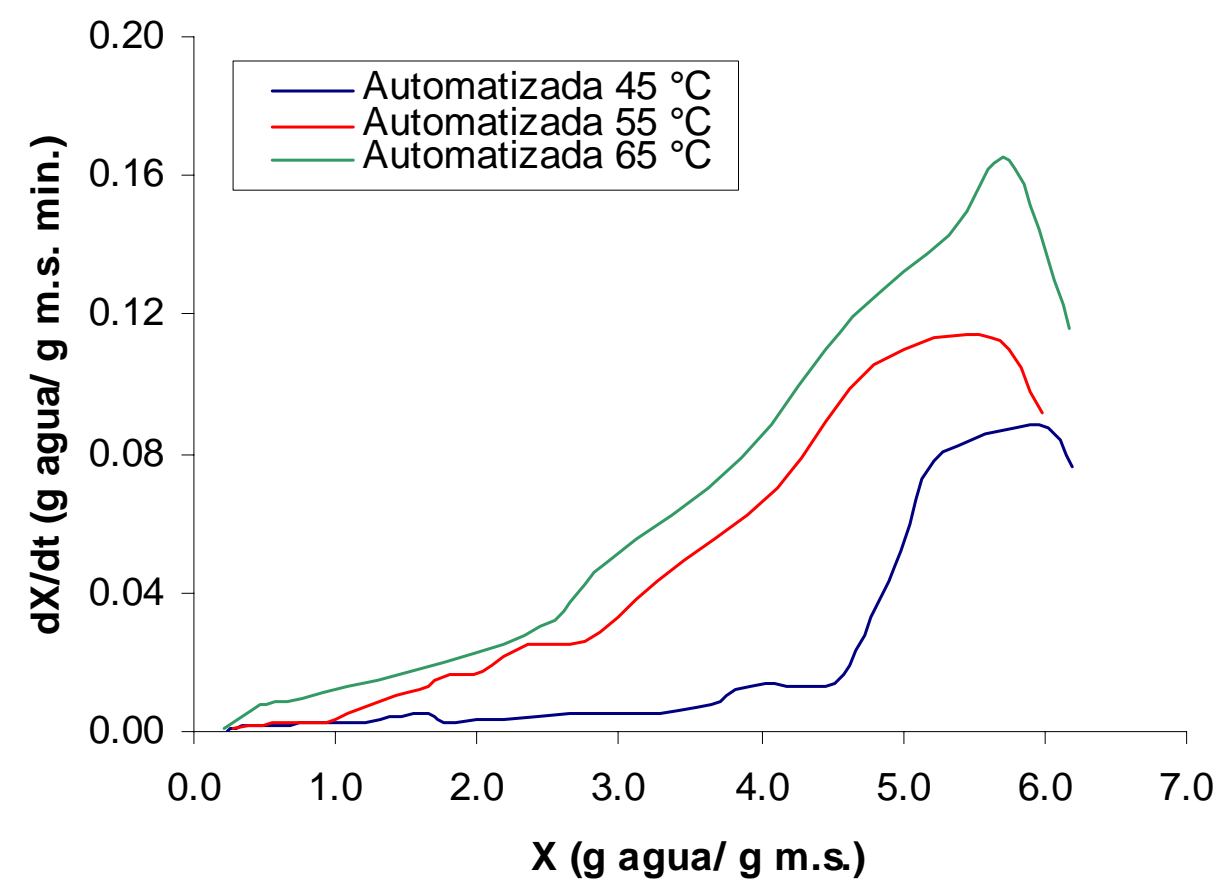

Fig. 5: Curvas de velocidad de secado automatizadas para cilindros de manzana a tres temperaturas

La experiencia realizada utilizando el dispositivo automático fue bien acogida por los usuarios alumnos en ambos laboratorios), ya que la interface resulto fácil de utilizar por parte de ellos, permitiendo participar a una mayor cantidad de alumnos, los cuales pudieron visualizar directamente a través del sistema de cámara web la evolución del proceso de secado que ocurrió al interior del equipo. Es importante destacar que de no contar con este tipo de dispositivos solo un número limitado de alumnos (operadores del equipo) podrían tener acceso directo al fenómeno.

Por otro lado la experiencia fue compartida en tiempo real por el grupo de alumnos en la ciudad de Valparaíso, los que, contando solo con una conexión de Internet pudieron visualizar el fenómeno y todas las variables del proceso implicadas. Así mismo, en forma directa pudieron requerir la información generada en el laboratorio de origen de modo de procesar dichos datos para análisis posteriores en profundidad.

\section{CONCLUSIONES}

El desarrollo de este prototipo permitió realizar experiencias de secado a distintas temperaturas de manera automática, prescindiendo de manipulación humana tanto de la muestra de alimento y como de las variables de proceso. Esto facilito el manejo y registro de los datos resultando en un proceso más flexible, expedito y con menos error asociado.

La interfaz visual desarrollada y disponible para múltiples usuarios en línea, facilito el proceso de enseñanza-aprendizaje por parte de alumnos de Ingeniería de Alimentos en dos laboratorios físicamente distantes, debido a su características mas atractivas y amistosas con el usuario (entorno Windows), mejorando el acceso a la fenomenología estudiada.

Finalmente se puede concluir que la propuesta de automatización es viable y valida, dado que los índices de validación utilizados (RMS, RSE X) presentaron valores adecuados para fines de docencia universitaria de pregrado. 


\section{AGRADECIMIENTOS}

A la Dirección de Investigación de la Pontifica Universidad Católica de Valparaíso y a la Dirección de Investigación y Desarrollo Académico de la Universidad Tecnológica Metropolitana por el apoyo otorgado para la realización de la presente investigación.

\section{REFERENCIAS}

A.O.A.C., "Official methods of analysis". Association of Official Analytical Chemists ( $N^{\circ} 920.151$ ). William Horwitz (ed), 13a ed., Washington(2005).

Fito, P. y otros tres autores; Introducción al Secado por Aire Caliente, 1ª Edición, 111-144. Editorial U.P.V., Valencia, España (2001).

Fito, P. y otros tres autores; Advanced food process engineering to model real foods and processes: The "SAFES" methodology, J. Food Engineering: 83(2), 173-185 (2007).

Harper, W.J., P.D. Courtney y G.W: Chism III; An Immersion Approach to Teaching Food Science, J. Food Science Education: 2(3), 53-56 (2003).

Nieto, A., y otros tres autores; Air drying behaviour of apples as affected by blanching and glucose impregnation, J. Food Engineering: 36(1), 63-79 (1998).

Nieto, A., M.A. Castro y S.M. Alzamora; Kinetics of moisture transfer during air drying of blanched and/or osmotically dehydrated mango, J. of Food Engineering: 50(3), 175-185 (2001).

Ogot, M., G. Elliot y N. Glumac; An Assessment of I-Person and Remotely Operated, J. Engineering Education: 92(1), 57-62 (2003)

Palou, E., y otros siete autores; Internet-Assisted Remote Laboratory Experiments, Computación Aplicada a la Industria de Procesos, CAIP 2003, 397, Puebla - México, 20 - 23 Octubre (2003).

Simal, S. y otros tres autores; Drying of shrinking cylinder-shaped bodies, J. Food Engineering: 37(4), 423-435 (1998).

Simpson, R.J., y otros tres autores, Simultaneous heat and mass transfer applied to non-respiring foods packed in modified atmosphere, J. of Food Engineering: 61(2), 279-286 (2004)

Tapia, C. y otros cinco autores; Development and Use of a Web Site with Multimedia Contents as a Complement to Traditional Unit Operations Courses, J. Food Science Education: 4(3), 41-46 (2005).

Vega, A., Andrés, A. y Fito, P. Modelado de la Cinética de Secado del Pimiento Rojo (Capsicum annuum L. CV Lamuyo). Rev. Información Tecnológica, ISSN 0718-0764 (en línea), vol.16 (6), 3-11, 2005. <http://www.scielo.cl/scielo.php?script=sci_arttext\&pid=S0718-07642005000600002\&lng=es\&nrm=iso>. Acceso: 7 de Enero (2008)

Wang, Z. y otros seis autores; Mathematical modelling on hot air drying of thin layer apple pomace, Food Research International: 40(1), 39-46 (2007).

Welti-Chanes, J. y otros siete autores; Food Engineering Education in Mexico, Central America, and South America, J. Food Science Education: 1(3), 59-65 (2002). 